Bull. Mater. Sci., Vol. 6, No. 6, December 1984, pp. 1059-1062. (C) Printed in India.

\title{
Spin-wave excitations in amorphous ferromagnets
}

\author{
ANIL K BHATNAGAR, B BHANU PRASAD, N RAVI and \\ R JAGANNATHAN* \\ School of Physics, ${ }^{*}$ School of Chemistry, University of Hyderabad, Hyderabad 500134 , India \\ MS received 7 May 1983
}

\begin{abstract}
Mössbauer measurements have been performed on a number of metallic glasses. The temperature dependence of average hyperfine or internal magnetic field $H_{\text {eff }}(T)$ arises from long wavelength spin wave excitations in these glasses. Values of $B_{3 / 2}$ and $C_{5 / 2}$ are in general much higher than those observed for crystalline ferromagnets indicating higher density of states for spin waves in amorphous ferromagnets.
\end{abstract}

Keywords. Spin wave excitations; amorphous ferromagnets; Mössbauer measurements; metallic glasses: temperature dependence.

\section{Introduction}

Amorphous metallic alloys, prepared by rapid quenching of molten alloys, have become an important class of materials from theoretical as well as practical points of view in recent years. There have been extensive studies of magnetic and electrical properties of iron-rich metallic glasses in the past decade. Study of magnetic phenomena in these glasses presents a fascinating field for theorists and experimentalists as magnetism is a structure sensitive property, and therefore, it is expected to be affected in amorphous solids. In this paper we discuss the temperature dependence of hyperfine magnetic field of iron-rich metallic glasses as measured by the ${ }^{57} \mathrm{Fe}$ Mössbauer spectroscopy. Results are interpreted in terms of excitations of long wavelength spin waves.

\section{Experimental}

Mössbauer spectra of a number of commercially available metallic glasses $\left(\mathrm{Fe}_{81} \mathrm{~B}_{13.5} \mathrm{Si}_{3.5} \mathrm{C}_{2}, \mathrm{Fe}_{78} \mathrm{~B}_{13} \mathrm{Si}_{9}, \mathrm{Fe}_{79} \mathrm{~B}_{16} \mathrm{Si}_{5}, \mathrm{Fe}_{40} \mathrm{Ni}_{40} \mathrm{~B}_{20}\right.$ and $\left.\mathrm{Fe}_{67} \mathrm{Co}_{18} \mathrm{~B}_{14} \mathrm{Si}_{1}\right)$ were recorded in the temperature range $77-900 \mathrm{~K}$ using an Elscint spectrometer coupled with a 256 channel MCA. The spectrometer was used in the constant acceleration mode and the source was ${ }^{57} \mathrm{Co}$ in rhodium matrix. The Curie temperatures of $\mathrm{Fe}_{81} \mathrm{~B}_{13.5} \mathrm{Si}_{3.5} \mathrm{C}_{2}, \mathrm{Fe}_{78} \mathrm{~B}_{13} \mathrm{Si}_{9}, \mathrm{Fe}_{40} \mathrm{Ni}_{40} \mathrm{~B}_{20}$ and $\mathrm{Fe}_{79} \mathrm{~B}_{16} \mathrm{Si}_{5}$ were determined by the constant Doppler velocity thermal-scan method (Bhanu Prasad et al 1983), while that of $\mathrm{Fe}_{67} \mathrm{Co}_{18} \mathrm{~B}_{14} \mathrm{Si}_{1}$ was estimated by plotting hyperfine magnetic field, $H_{\mathrm{eff}}(T) v \mathrm{~s}$ temperature since the Curie temperature, $T_{c}$, in this case was larger than the crystallization temperature (Bhanu Prasad et al 1980). $H_{\text {eff }}(T)$ values were determined by fitting each spectrum to the Lorentzian line-shape sextet pattern. The spectrometer was calibrated using a thin iron foil. 


\section{Results and discussion}

The decrease in magnetization of a crystalline ferromagnet at low temperatures can be explained if the low lying magnetic excitations are spin waves which obey the following dispersion relation:

$$
\hbar w(q)=D q^{2}+E q^{4}+\ldots
$$

where $h w(q)$ is the energy of spin wave with propagation wave number $q$, and $D$ and $E$ are the stiffness constants. Usually $E$ is much smaller than $D$. This dispersion relation leads to the following temperature dependence of magnetization $M(T)$,

$$
M(T)=M(O)\left(1-B T^{3 / 2}-C T^{5 / 2}-\ldots\right),
$$

where $B$ and $C$ are constants and are related to $D$ and $E$ as given below.

$$
\begin{aligned}
& B=2.612\left[g \mu_{B} / M(O)\right]\left(k_{B} / 4 \pi D\right)^{3 / 2} \\
& C=1.341\left[g \mu_{B} / M(O)\right]\left(k_{B} / 4 \pi D\right)^{5 / 2}(15 \pi E / D)
\end{aligned}
$$

where $k_{B}$ is the Boltzmann constant, $\mu_{B}$ is the Böhr magneton and $g$ is the usual $g$ factor. Equation 2 can be rearranged to give

$$
\frac{M(T)-M(O)}{M(O)}=-B T^{3 / 2}-C T^{5 / 2}-\ldots
$$

or

$$
\frac{M(T)-M(O)}{M(O)}=-B_{3 / 2}\left(T / T_{c}\right)^{3 / 2}-C_{5 / 2}\left(T / T_{c}\right)^{5 / 2}-\ldots
$$

Inelastic neutron scattering measurements on some of amorphous ferromagnets have established the existence of long wavelength spin waves obeying the dispersion relation given by (1) (Lynn et al 1976; Rhyne et al 1979). Similarly magnetization measurements (Tsuei et al 1968; Kaul 1981) and ferromagnetic resonance studies (Spano and Bhagat 1981) have shown that the temperature dependence of $M(T)$ follows the behaviour predicted by (2).

From Mössbauer measurements the average internal magnetic field $H_{\text {eff }}(T)$ is determined. It has been found experimentally that for most iron-rich metallic glasses, the average internal magnetic field is directly proportional to the magnetization to a good approximation and the proportionality constant is approximately $135 \mathrm{kOe} / \mu_{B}$ (Chien et al 1979). Therefore, (5) and (6) can be expressed in terms of $H_{\mathrm{eff}}(T)$ as follows:

$$
\frac{H_{\mathrm{eif}}(T)-H_{\mathrm{eff}}(O)}{H_{\mathrm{eff}}(O)}=-B T^{3 / 2}-C T^{5 / 2}-\ldots
$$

or

$$
\frac{H_{\mathrm{eff}}(T)-H_{\mathrm{eff}}(O)}{H_{\mathrm{eff}}(O)}=-B_{3 / 2}\left(T / T_{\mathrm{c}}\right)^{3 / 2}-C_{5 / 2}\left(T / T_{\mathrm{c}}\right)^{5 / 2}-\ldots
$$

where $B, C, B_{3 / 2}$, and $C_{5 / 2}$ constants remain the same as in (5) and (6). Thus the temperature dependence of $H_{\text {eff }}(T)$ at low temperatures can also be interpreted in terms of excitations of spin waves. At first it may seem surprising that spin waves can be excited in noncrystalline solids. However, the situation is quite akin to the sound wave 
propagation in glasses. This is so because the long wavelength modes are not sensitive to the local order or disorder. In particular Herring and Kittel (1951) have shown that long wavelength spin waves can exist even in a ferromagnetic continuum medium.

Average hyperfine magnetic fields of various metallic glasses were determined as explained in $\S 2$. A plot of $\left[H_{\mathrm{eff}}(T)-H_{\mathrm{eff}}(O)\right] / H_{\mathrm{eff}}(O) v s\left(T / T_{c}\right)^{3 / 2}$ for various metallic glasses is shown in figure 1 . A computer least square fit of (7) and (8) to experimental data yields values of $B, C, B_{3 / 2}$ and $C_{5 / 2}$ which are given in table 1 . For sake of comparison, the values of these constants for crystalline $\mathrm{Fe}$ and $\mathrm{Ni}$, and glassy $\mathrm{Fe}_{40} \mathrm{Ni}_{40} \mathrm{P}_{14} \mathrm{~B}_{6}, \mathrm{Fe}_{80} \mathrm{~B}_{20}$ and $\mathrm{Fe}_{82} \mathrm{~B}_{12} \mathrm{Si}_{6}$ are also listed in the table. It is observed that $B_{3 / 2}$ values range from $0 \cdot 2-0.4$ and are much larger than the values for crystalline $\mathrm{Fe}$ and Ni. Also $T^{3 / 2}$ dependence of relative change in $H_{\text {eff }}(T)$ extends to much higher temperatures than observed for crystalline ferromagnets. $T^{3 / 2}$ plus $T^{5 / 2}$ dependence, as given by (7) and ( 8 ), can be fitted to experimental data over a large temperature interval. Values of $B\left(B_{3 / 2}\right)$ and $C\left(C_{5 / 2}\right)$ depend upon the temperature interval used to fit the data (Bhatnagar et al 1982; Saegusa and Morrish 1982). If data is fitted over a large temperature interval then it has the effect of reducing the $B\left(B_{3 / 2}\right)$ value and increasing the $C\left(C_{5 / 2}\right)$ value which is expected. It has also been observed that $B$ and $C$ values

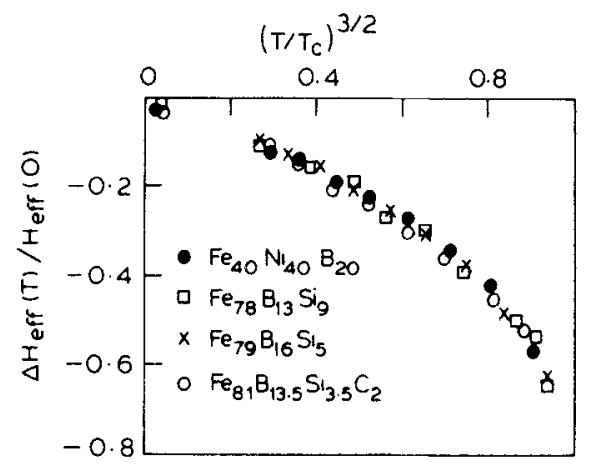

Figure 1. Plot of $\left[H_{\mathrm{eff}}(T)-H_{\mathrm{eff}}(O)\right] / H_{\mathrm{eff}}(O)$ vs $\left(T / T_{\mathrm{c}}\right)^{3 / 2}$ for various iron-rich metallic glasses.

Table 1. Sample compositions, Curie temperature $T_{c}$, coefficients $B, C, B_{3 / 2}$ and $C_{5 / 2}$ for amorphous ferromagnets and crystalline $\mathrm{Fe}$ and $\mathrm{Ni}$.

\begin{tabular}{|c|c|c|c|c|c|c|}
\hline System & $T_{c}(\mathbf{K})$ & $B\left(10^{-6} \mathrm{~K}^{-3 / 2}\right)$ & $C\left(10^{-8} \mathrm{~K}^{-5 / 2}\right)$ & $B_{3 / 2}$ & $C_{5 / 2}$ & Reference \\
\hline $\mathrm{Fe}_{80} \mathrm{~B}_{20}$ & 685 & 22 & 12 & 0.40 & 0.17 & Chien (1978) \\
\hline $\mathrm{Fe}_{40} \mathrm{Ni}_{40} \mathrm{~B}_{20}$ & 695 & 22 & 0.4 & 0.40 & 0.06 & Present work \\
\hline $\mathrm{Fe}_{40} \mathrm{Ni}_{40} \mathrm{P}_{14} \mathrm{~B}_{6}$ & 537 & 38 & 1.2 & 0.47 & 0.08 & Chien and Hasegawa (1977) \\
\hline $\mathrm{Fe}_{78} \mathrm{~B}_{13} \mathrm{Si}_{9}$ & 733 & 10 & $2 \cdot 4$ & $0 \cdot 21$ & $0-35$ & Present work \\
\hline $\mathrm{Fe}_{79} \mathrm{~B}_{16} \mathrm{Si}_{5}$ & 730 & 11 & $2 \cdot 3$ & 0.22 & $0 \cdot 33$ & Present work \\
\hline $\mathrm{Fe}_{82} \mathrm{~B}_{12} \mathrm{Si}_{6}$ & 658 & - & - & 0.34 & 0.21 & Ok and Morrish (1980) \\
\hline $\mathrm{Fe}_{81} \mathrm{~B}_{13.5} \mathrm{Si}_{3.5} \mathrm{C}_{2}$ & 698 & 16 & $2 \cdot 1$ & $0 \cdot 30$ & 0.27 & Present work \\
\hline $\mathrm{Fe}_{81} \mathrm{~B}_{13.5} \mathrm{Si}_{3.5} \mathrm{C}_{2}$ & 668 & $\ldots$ & - & 0.30 & 0.29 & Saegusa and Morrish (1982) \\
\hline Crystalline $\mathrm{Fe}$ & 1042 & 3.4 & $0 \cdot 1$ & $0 \cdot 114$ & 0.04 & Chien and Hasegawa (1977) \\
\hline Crystalline $\mathrm{Ni}$ & 637 & 7.5 & 1.5 & 0.117 & 0.15 & Chien and Hasegawa (1977) \\
\hline
\end{tabular}


become much smaller after the amorphous material crystallizes (Hasegawa and Ray 1979). Hence, the high $B\left(B_{3 / 2}\right)$ and $C\left(C_{5 / 2}\right)$ values seem to be the characteristic of the amorphous state. Higher $B$ values in glassy ferromagnets also mean lower values of $D$, the stiffness constant, for glassy systems as seen from (3). Lower values of $D$ also mean that the density of states for spin waves are higher in the amorphous state. Theoretical calculations do show higher density of states for spin waves in amorphous materials due to fluctuations in the exchange interaction arising from the random atomic arrangement (Montgomery et al 1970; Simpson 1974). Thus the existence of spin wave excitations in amorphous ferromagnets is clearly demonstrated by Mössbauer measurements as well as by magnetization (Kaul 1981) and inelastic neutron scattering measurements (Lynn et al 1976; Axe et al 1977; Rhyne et al 1979).

\section{Conclusion}

The temperature dependence of the average internal magnetic field of a number of ferromagnetic metallic glasses can be described by the expression $H_{\text {eff }}(T)=H_{\text {eff }}(O)(1$ $\left.-B T^{3 / 2}-C T^{5 / 2}-\ldots\right)$, which is indicative of spin wave excitations in these solids. Values of $B\left(B_{3 / 2}\right)$ are much higher than those for crystalline $\mathrm{Fe}, \mathrm{Ni}$ etc. This observation can be explained if higher density of states for spin waves are assumed in the amorphous ferromagnets. Values of $B\left(B_{3 / 2}\right)$ and $C\left(C_{5 / 2}\right)$ are found to depend upon the temperature interval used to fit experimental data to the $T^{3 / 2}$ and $T^{5 / 2}$ dependence.

\section{Acknowledgements}

Authors thank the Science and Engineering Research Council (DST, India) and the Department of Atomic Energy for support.

\section{References}

Axe J D, Shirane G, Mizoguchi T and Yamauchi K 1977 Phys. Rev. B15 2763

Bhanu Prasad B, Bhatnagar A K and Jagannathan R 1980 Solid State Commun. 36661

Bhanu Prasad B, Bhatnagar A K and Jagannathan R 1983 J. Appl. Phys. 542019

Bhatnagar A K, Bhanu Prasad B, Ravi N, Jagannathan R and Anantharaman T R 1982 Solid State Commun: 44905

Chien C L 1978 Phys. Rev. B18 1003

Chien C L and Hasegawa R 1977 Phys. Rev. B16 2115

Chien C L, Musser D, Gyorgy E M, Sherwood R C, Chen H S, Luborsky F E and Walter J L 1979 Phys. Rev. B20 283

Hasegawa R and Ray R 1979 Phys. Rev. B20 211

Herring C and Kittel C 1951 Phys. Rev. 81869

Kaul S N 1981 Phys. Rev. B24 6550

Lynn J W, Shirane G, Birgeneau R J and Chen H S 1976 AIP Conf. Proc. 34313

Montgomery C G, Krugler J I and Stubbs R M 1970 Phys. Rev. Lett. 25669

Ok H N and Morrish A H 1980 Phys. Rev. B22 4215

Rhyne J J, Lynn J W, Luborsky F E and Walter J L 1979 J. Appl. Phys. 501583

Saegusa N and Morrish A H 1982 Phys. Rev. B26 10

Simpson A W 1974 Wiss Z. dTH Dresden 31020

Spano M L and Bhagat S M 1981 J. Magn. Mag. Mater. 24143

Tsuei C C, Longworth G and Lin S C H 1968 Phys. Rev. 170603 